\title{
Time-varying Acoustic Emission Characterization for Guidewire Coronary Artery Perforation Identification
}

\author{
Alfredo Illanes, Anna Schaufler, Iván Maldonado, Axel Boese, Michael Friebe \\ INKA Intelligente Katheter, Otto von Guericke University, Magdeburg, Germany
}

\begin{abstract}
Artery guidewire-induced perforation during coronary interventions is an uncommon but potentially serious complication with significant morbidity and mortality rates. For minimizing its impact it is crucial for the surgeon to early detect that a perforation has occurred. However, this is not always easy since perforation sometimes is not characterized by any symptom or sign. In this work a timevarying (TV) characterization of coronary artery perforation is proposed through a TV parametrical modeling of an audio signal acquired from the proximal part of a guide wire. Results show that guide wire perforation leaves a characteristic TV trace which can be tracked through the $T V$-poles and spectrum. This results in clear differentiating patterns allowing around $90 \%$ of correct perforation classification.
\end{abstract}

\section{Introduction}

The use of medical interventional devices (MIDs) such as needles, catheters and guidewires is today a common procedure in medicine for performing minimally invasive therapy and diagnosis $[1,2]$. Generally in all these type of procedures the operator needs to introduce the MID inside the body and place it into a target and/or follow a determined path without damaging important structures or organs.

Particularly for performing minimally invasive therapies for treating endovascular diseases, catheters and guide wires that are controlled externally by an interventionist are used. A guide wire consists on a small wire that can be placed inside a catheter to allow steering of the catheter through the vasculature [3].

The perforation of vessel walls during endovascular procedures is a rare but risky complication. For percutaneous coronary interventions the rate of occurrence is between $0.1 \%$ and $4.0 \%$ [4-6]. There is a wide range of possible consequences of perforations, from clinically irrelevant aftereffects that can be mitigated well with conservative therapy, to cardiac tamponade that can provoque the death of the patient $[4,5]$.
The consequences of a perforation can be treatable, however the outcome of such a treatment are highly dependent on the early detection that a perforation has occurred. However, this is not always easy since perforation sometimes is not characterized by any symptom or sign.

Several approaches has been proposed for acquiring guidance information from guidewires. Most of them are based on measuring force using piezoresistive sensors with the goal of acquiring haptic information from the guide wire [7]. Other works have proposed piezoelectric and optical sensors for measuring pressure [8] and piezoelectric transducers for measuring blood flow velocity for monitoring vessel occlusion $[9,10]$. The main drawback of the proposed approaches are that sensors are usually located at the distal part of the guide wire, i.e., invasively in the part of the device that is inserted inside the body.

In this work a time-varying (TV) characterization of coronary artery perforation is proposed through a TV parametrical modeling of an audio signal acquired with a stethoscope connected to the proximal end of a guide wire. The main idea behind this new approach is to classify an occurring guide wire event as being a vessel perforation or artifact by analyzing the time-varying dynamics of the acquire audio signal during guide wire insertion.

A database with recordings belonging to perforation and to different generated artifacts has been implemented to evaluate the performances of the new approach. Results show that a vessel perforation leave a clear trace on the audio signal that it is possible to characterize through several parameters using time-varying parametrical modeling. This characterization serves for differentiating perforation from artifacts opening perspectives for early detection of vessel perforation during minimally invasive procedures.

\section{Materials and methods}

\subsection{Experimental setup and database im- plementation}

A stethoscope equipped with a microphone connected to a smart phone has been firmly attached to the proximal 
(a)

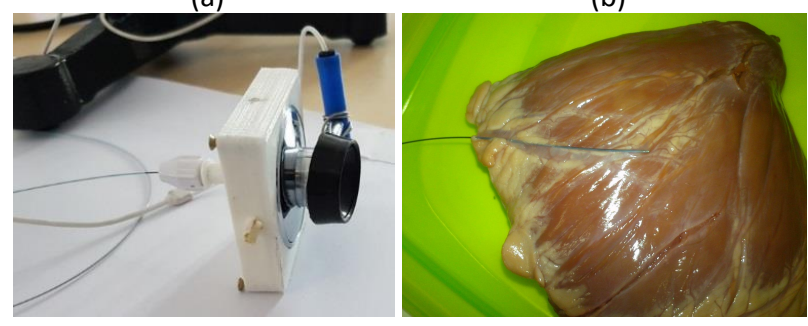

Figure 1. a) view of the guidewire attached to a stethoscope connected to a microphone, b) guide wire inserted in a pork heart vessel.

end of a 0.014 -inch guide wire via a 3D printed adapter allowing a direct contact with the stethoscope membrane (see Figure 1a).

Coronary arteries belonging to 4 pork hearts were perforated using the tip of the guide wire (see Figure 1b). During the procedure audio signals were recorded in MP3 format with a sampling frequency of $44800 \mathrm{~Hz}$ and the time instant at which the perforation occurred has been annotated.

An audio database has been implemented with the acquired audio recordings in order to evaluate the performances of the proposed approach. It included 100 coronary artery perforations audio recordings, each one with a duration of 30 seconds. In order to perform classification between perforation and non-perforation the database included also 200 additional recordings with different types of induced guidewire audio artifacts, including friction between the guidewire and the artery wall and tiny guidewire bumps.

\subsection{Audio signal feature extraction and classification}

The block diagram of Figure 2 shows the main steps for classifying audio recordings as perforations or artifact. Each audio signal has been first decimated and then filtered using a wavelet based band-pass filter. The resulting signal has been modeled using a time-varying autoregressive (TV-AR) parametrical model for estimating a time-varying (TV) power spectral density and TV poles. Finally different features has been computed from the AR spectrum and from the maximal energy AR pole.

\subsubsection{Audio signal preprocessing}

As a first step the audio signal has been decimated by 10 and then the resulting signal was bandpass filtered using Discrete Wavelet Transform (DWT). For that the signal has been decomposed in 10 scales using a Daubechies DWT and finally reconstructed with selected middle-frequency wavelet scales [11].

\subsubsection{Time-varying AR modeling}

The features used for classification between perforation and artifact are computed from a TV-AR model of the audio signal. Lets $y[n]$ being the decimated and preprocesssed audio signal. An AR model assumes that the value of the current sample $y[n]$ at sample number $n$ can be modeled as a linearly weighted sum of the $p$ most recent sample values, and a white zero mean noise $e[n]$. Since the audio signal during a perforation is highly non-stationary, the AR model has to be made time-varying and therefore the weights (AR parameters) have to be time dependent, resulting in the TV parametrical model:

$$
y[n]=-\sum_{k=1}^{p} a_{k}(n) y[n-1]+e[n]
$$

where $p$ is the model's order and $a_{k}(n)\{k=1,2, \ldots, p\}$ are the TV-AR parameters. Equation (1) give rise to a timevarying spectrum

$$
S_{A R}(f, n)=\frac{1}{\left|1+\sum_{k=1}^{p} a_{k}(n) e^{j 2 \pi f n}\right|^{2}}
$$

and to a time-varying pole representation

$$
H(z, n)=\frac{1}{\prod_{k=1}^{p}\left(1-z_{k}(n) z^{-1}\right)} .
$$

In this work the TV-AR model has been computed over a sliding window of $50 \mathrm{~ms}$ width and an overlap of $95 \%$. In each window a $20^{\text {th }}$ order AR model has been used to estimate the AR parameters using the Yule-Walker method and for each of the windows the AR spectrum and poles were computed.

The TV-pole representation of equation (3) is used for computing the evolution of the maximal energy pole, i.e., the pole that, at each time window, has the maximal spectral power. It is computed by calculating first the $r$ time dependent resonant frequencies from the phase angle, $\theta_{k}(n)$, of the corresponding pole in the upper half of the complex plane [12]:

$$
f_{k}(n)=\frac{\theta_{k}(n)}{2 \pi}=\tan ^{-1}\left(\frac{\operatorname{Im}\left(z_{k}(n)\right)}{\operatorname{Re}\left(z_{k}(n)\right)}\right) \frac{f_{s}}{2 \pi}
$$

where $f_{s}$ and $f_{k}, k=1,2, \ldots, r$ correspond to the sampling frequency and to the $r$ resonant frequencies resulting from the poles, respectively. Then the spectral power $P_{k}$ of the resonant frequency $k$ is obtained from the real part of the residue term $r_{k}$ :

$$
\begin{aligned}
r_{k}(n) & =\left.z^{-1}\left(z-p_{k}(n)\right) H(z, n)\right|_{z=z_{k}(n)} \\
P_{k}(n) & =\left.2 \sigma^{2} \operatorname{Re}\left(r_{k}(n)\right)\right|_{z=z_{k}(n)} .
\end{aligned}
$$




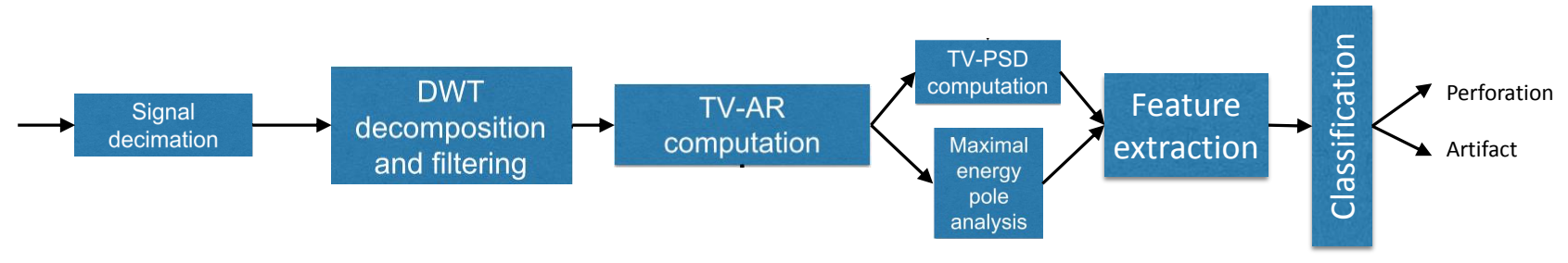

Figure 2. Main steps of the signal processing algorithm for perforation feature extraction and classification.

Finally at each time instant $n$ the maximal energy pole has been computed as the frequency belonging to the pole having the maximal power from the $r$ resonant frequencies.

\subsubsection{Feature extraction and classification}

The features used for classifying between artery perforation and artifact are computed from both the TV-AR spectrum and the TV-pole representation in order to extract a trace in the audio signal that can characterize patterns of the interaction between the guide wire tip and the vessel wall.

Figure 3 illustrate four real cases used to explain the main idea behind the features that are used for perforation classification. It shows one second audio signal segments together with their respective TV-AR spectrums and maximal energy pole signals. Figures $3 \mathrm{a}$ and $\mathrm{b}$ display cases belonging to two different pork heart perforations while Figures $3 \mathrm{c}$ and d display cases where a friction and a guide wire bump respectively have occurred.

Looking at these figures it is possible to verify the following:

- The TV-AR spectrum shows main frequency components that are stable in time during a perforation (inside the segment of higher energy in the TV spectrum), unlike an artifact where the frequencies are more disperse in time. - During a perforation some significant segments can be distinguished in the maximal energy pole indicator: 1) a strong overshoot represented by a very fast and short rice of the pole frequency, 2) a plateau just after the overshoot until the signal goes back to a stationary stage. It is possible to observe that the overshoot is not present when an artifact occurs and also that the plateau is much more stable when a perforation occurs than when an artifact is generated.

This analysis leads us to consider three main features:

- Presence or absence of a significant overshoot at the beginning of the event.

- Level of stability of the plateau in terms of high or low variability.

- Stability of the main frequency components in time through an indicator of correlation between spectrums belonging to different time windows.
Finally for classifying between perforation or artifact, a simple thresholding step is applied over a final indicator computed by a product operation between the three obtained features.

\section{Results}

Figure 4 shows the final indicator value (normalized between 0 and 1) for the 300 audio recordings of the database. The first 165 recordings belong to friction, the recordings going from nb. 166 to the nb. 265 belong to heart coronary artery perforations and the rest belong to guide wire bumps.

It is clearly identifiable that the indicator shows in average a higher value for perforations than for artifacts. This is explained by the fact that perforations are characterized by presence of overshoot, stability of plateau and stability of the main frequency components compared to the generated artifacts. By thresholding the indicator in $0.43,89.5 \%$ of perforation can be classified.

\section{Conclusions}

In this work a new approach for characterizing a puncture using non-invasive sensoring has been presented. Audio signals acquired with a stethoscope connected to the proximal end of a guide wire has shown significant signal dynamic characteristics that can allow a correct classification with a simple indicator and without the use of any complex intelligent technique. Preliminary results show that the time-varying trace that a perforation leaves in the audio signal is significantly different than possible artifacts that may occur during an intervention.

\section{Acknowledgements}

This research was funded by the German BMBF (grant 03IPT7100X).

\section{References}

[1] Abolhassani N, Patel R, Moallem M. Needle insertion into soft tissue: A survey. Medical engineering physics 2007; 29(4):413-431. 


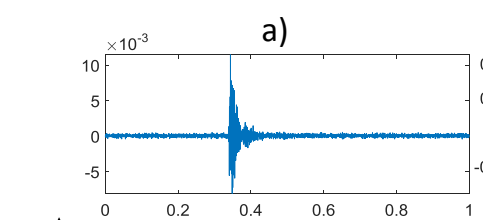

b)
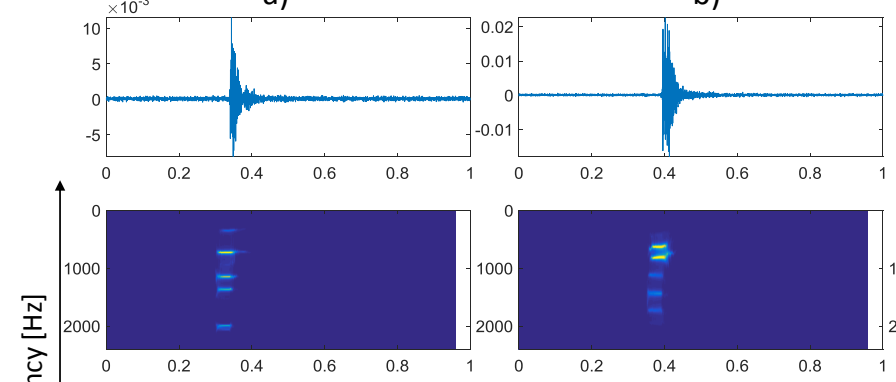

c)
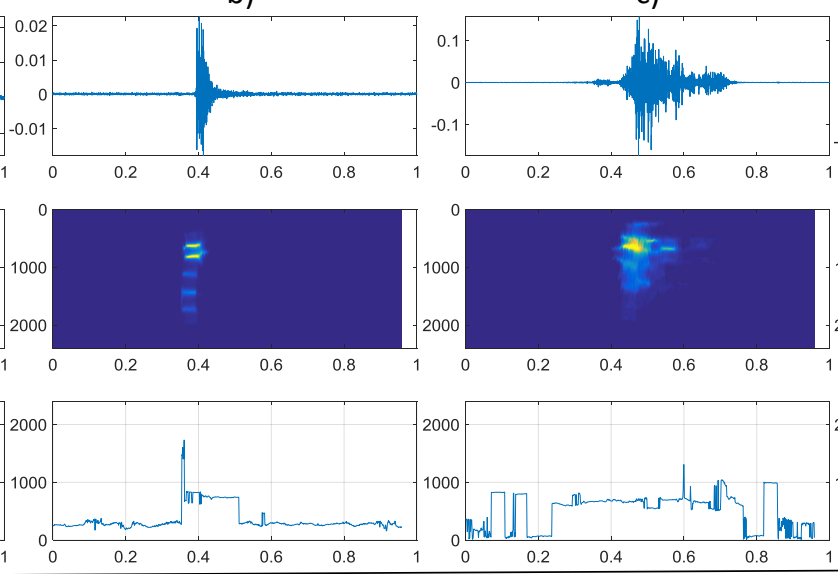

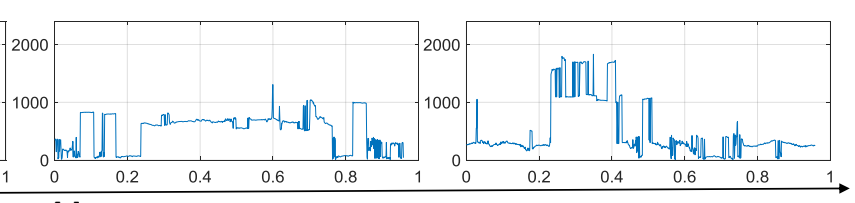

Time $[s]$

Figure 3. Audio signals with the respective TVAR spectrums and maximal energy pole signals for four real cases. a) and b) show cases where a pork heart perforation has occurred for two different pork hearts and c) and d) show cases where an artifact has occurred; a friction in c) and a guide wire bump in d).

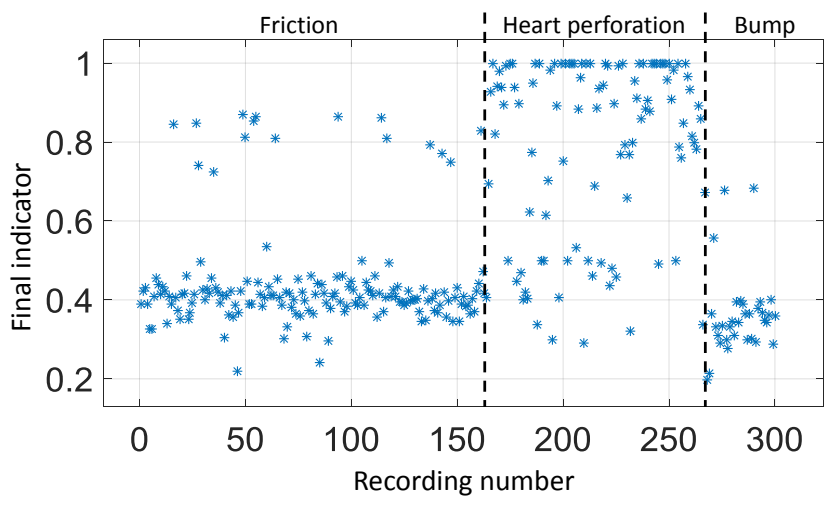

Figure 4. Final indicator values for the whole database, based on the extracted features.

[2] Stoll A, van Oepen A, Friebe M. Intraoperative delivery of cell-killing boost radiation-a review of current and future methods. Minimally Invasive Therapy Allied Technologies 2016;25(4):176-187.

[3] Boese A. Catheters in vascular therapy. Medical Devices 2013;99-117.

[4] Teis-Soley A, Fernández-Nofrerías E, Rodríguez-Leor O, Tizón H, Salvatella N, Valle V, Mauri J. Coronary artery perforation by intracoronary guidewires: Risk factors and clinical outcomes. Revista Espanola de Cardiologia English Edition 2010;63(6):730-734.

[5] Gunning M, Williams I, Jewitt D, Shah A, Wainwright R, Thomas M. Coronary artery perforation during percutaneous intervention: incidence and outcome. Heart 2002; 88(5):495-498.

[6] Guo R, Yang L. The successful use of autologous skin in management of guidewire-induced distal coronary perforation. Clinical case reports 2017;5(6):1018-1021.

[7] Opitz T, Neupert C, Werthschützky R. Development of a haptic assistive system for force transmission onto a guide wire. In Actuator-13th International Conference on New Actuators. 2012; 620-623.

[8] Kern M. Comparing ffr tools: New wires and a pressure microcatheter. Cathet Lab Digest 2016;24:4-9.

[9] Okamura A, Ito H, Iwakura K, Kawano S, Inoue K, Maekawa Y, Ogihara T, Fujii K. Detection of embolic particles with the doppler guide wire during coronary intervention in patients with acute myocardial infarction: efficacy of distal protection device. Journal of the American College of Cardiology 2005;45(2):212-215.

[10] Ferrari M, Werner GS, Bahrmann P, Richartz BM, Figulla HR. Turbulent flow as a cause for underestimating coronary flow reserve measured by doppler guide wire. Cardiovascular ultrasound 2006;4(1):14.

[11] Illanes A, Haritopoulos M. Fetal heart rate feature extraction from cardiotocographic recordings through autoregressive model's power spectral-and pole-based analysis. In Engineering in Medicine and Biology Society (EMBC), 2015 37th Annual International Conference of the IEEE. IEEE, 2015; 5842-5845.

[12] Thanagasundram S, Spurgeon S, Schlindwein FS. A fault detection tool using analysis from an autoregressive model pole trajectory. Journal of Sound and Vibration 2008; 317(3):975-993.

Address for correspondence:

Alfredo Illanes

Rötgerstraße 9, 39104 Magdeburg, Germany

alfredo.illanes@ovgu.de 